\title{
Research on Remediation Methods of Contaminated Land and Development Trend
}

\author{
Daiwen Zhu ${ }^{1,2,3,4^{*}}$ \\ ${ }^{1}$ Shaanxi Provincial Land Engineering Construction Group Co., Ltd. Xi'an 710075, China; \\ ${ }^{2}$ Institute of Land Engineering and Technology, Shaanxi Provincial Land Engineering Construction \\ Group Co., Ltd. Xi'an 710075, China; \\ ${ }^{3}$ Key Laboratory of Degraded and Unused Land Consolidation Engineering, the Ministry of Land \\ and Resource, Xi'an 710075, China; \\ ${ }^{4}$ Shaanxi Provincial Land Consolidation Engineering Technology Research Center, Xi'an 710075, \\ China. \\ *zhudaiwen@163.com
}

Keywords: Soil pollution, Remediation, Development trend.

Abstract: The land pollution in China is becoming more and more serious. Soil pollution has brought damages to farmers' economic and health benefits. Therefore, it is particularly important to make use of technical methods to turn contaminated land into available land. At present, soil pollution can be remediated gradually by physical, chemical and biological remediation. In this paper, the remediation methods for contaminated land is discussed and summarized. The repaired principles, research progress and application scope of various methods are reviewed. Finally, the development trend of contaminated land restoration industry is proposed in China.

Original article, Published date: 2018-05-01

DOI: 10.23977/erej.2018.21001

ISSN: ISSN 2616-3756

https://www.clausiuspress.com/journal/LANDRM.html

\section{Introduction}

In recent years, with the increase in quality and safety issues of agricultural products and group health hazards caused by soil pollution, the threats to the food chain, public health, and ecological security have gradually attracted the attention of academics and political circles, and have also 
become focus of social concern. More attention has been paid to land security. In recent years, many scholars have given higher attention to soil pollution. However, In China, the pollution remediation method has not formed a complete system. The pollution land management concept is backward. The rules and regulations are not perfect, and the repaired method for soil pollution is single. In order to reduce the environmental pollution and economic losses caused by polluted soil, it is urgent to explore and improve the theory of contaminated land remediation so as to guide soil remediation engineering practice.

\section{Remediation methods}

\subsection{Physical method}

Physical technique is the technique of removing or separating contaminants (especially organic contaminants) from soil through various physical processes [1]. This method includes soil replacement methods, soil barrier landfill technique, and thermal desorption technologies and so on.

Replacement method is an effective physical technique to deal with brownfield by deep-flooding the degraded land to the bottom, and then covering clean soil on brownfield or by replacing pollution land with clean land directly. Replacement methods can effectively block the brownfield from the ecosystem, thereby reducing its impact on the environment. However, due to the large amount of work and high cost, this method is only suitable for small areas and serious soil pollution [2].

Soil barrier landfill technique is a method to place contaminated soil in the impervious-resistance landfill, and to block the migration and diffusion of contaminants in the soil through the barrier layer. In this way, contaminated soil is isolated from the surrounding environment to avoid contact of pollutants with human body and to avoid it from migration with precipitation or groundwater, thus causing harm to the human body and the surrounding environment. Contaminants come in contact with humans and migrate with precipitation or groundwater, causing harm to the human body and the surrounding environment. According to its implementation, it can be divided into in-situ and ex-situ barrier landfill techniques.

The in-situ barrier landfill is to build a barrier layer around the contaminated area and to cover the contaminated area with a barrier layer to completely isolate the perimeter and the top of the contaminated area from the surroundings. Based on the actual situation of the site and the results of risk assessment, a barrier is built only around the contaminated site or only covering the roof site. The ex-situ barrier landfill is to bury contaminated soil into a seepage barrier landfill consisting of high-density polyethylene and other barrier materials to isolate the contaminated soil from the surrounding environment and to prevent contaminants in the contaminated soil from migrating into precipitation or groundwater.

Thermal desorption technique is the process in which organic contaminants in the soil are heated by direct or indirect heat exchange. The content of organic contaminants in the soil is high enough to evaporate and separate from the soil mass. It is divided into in-situ and ex-situ thermal desorption techniques [3]. The In-situ thermal desorption is an important method for the remediation of oil contaminated soil, which is mainly used to deal with some areas that are more difficult to carry out ex-situ remediation such as the remediation for deep soil or soil under the building. This technique could heat up the soil to reach the volatilization temperature of the contaminants, and then extract it to the ground by vacuum extraction, and finally, discharge it after harmless treatment. The thermal desorption process can cause physical and chemical changes such as volatilization and cracking of organic compounds in the soil. Under the conversion of contaminants into a gas state, their fluidity will be greatly improved, and the volatilized products will be purified after being collected and captured. The ex-situ thermal desorption technology heats the contaminated soil to above the 
boiling point of the target contaminant by direct or indirect heating. By controlling the residence time of the material and the system temperature, it can selectively promote the gasification and volatilization of pollutants and target pollutants.

\subsection{Chemical remediation}

The chemical remediation method has developed earlier, mainly including soil solidification-stabilization, leaching and oxidation-reduction technology. The solidification-stabilization technology could fix pollutants in contaminated media to stable for a long period of time. It is divided into in-situ and ex-situ stabilization technologies [4]. The in-situ stabilization technology uses a certain mechanical force to add stabilizing agent to the contaminated medium in-situ. Thorough mixing sufficiently, this process causes physical and chemical effects to the contaminated medium and contaminants. The contaminated soil could be fixed as a solid structure with low permeability or pollutants that were converted into inactive chemical forms, reducing the migration and diffusion of pollutants in the environment.

The ex-situ stabilization treatment, however, can handle inorganic substances such as metals, asbestos, radioactive substances, corrosive inorganic substances, cyanide and arsenic compounds, pesticides, petroleum, polycyclic aromatic hydrocarbons, polychlorinated biphenyls, dioxins. However, this treatment could not be applied in VOCs remediation or projects that have acceptance targets for total pollutants.

The chemical oxidation-reduction technique is a method to add oxidizing agents (permanganate, hydrogen peroxide, Fenton's reagent, persulfate, ozone and etc.) or reducing agents (sodium dithionite, sodium bisulfite, ferrous sulfate, calcium polysulfide, divalent iron, zero-valent iron) into the contaminated soil [5]. Through oxidation or reduction remediation, soil contaminants could be converted into non-toxic or relatively less toxic substances.

The leaching remediation technology involves injecting water or an aqueous solution containing a washing aid, an acid and alkali solution, a complexing agent or a surfactant into the contaminated soil to elute and clean the contaminants in the soil or sediment. The in-situ method is to leach out the soil through injection wells [6]. The ex-situ leaching technology is using physical elution method to separate heavy contaminated soil components or transfer contaminants from the soil phase to the liquid phase by adding water or a suitable synergist. After the elution treatment, the amount of contaminated soil can be effectively reduced. This method should not be used in soils where the content of fine particle (clay and silt particle) is higher than $25 \%$.

Solvent extraction technique is using solvents to separate and remove sludge, sediment, and hazardous organic pollutants in the soil [7]. Since solvent extraction can effectively remove hazardous pollutants in the polluting medium, a part of the contaminants extracted or concentrated from the soil that has a certain economic value. Accordingly, the non-utilizable part can be correspondingly eliminated. Because the solvent extraction technology does not destroy the structure of the contaminants during operation, it can maximize the resource and value of the contaminants. At the same time, the solvent used in the extraction process can also be regenerated and reused. Therefore, it can be said the solvent extraction technology is a sustainable remediation.

\subsection{Bioremediation Technology}

The use of plants, animals, and microorganisms to absorb and convert contaminants in the soil to reduce its risk to acceptable levels or to convert contaminants into harmless forms can be classified as bioremediation. Under this concept, bioremediation can be divided into three types: phytoremediation, animal remediation and microbial remediation. At present, animal remediation 
technology mainly utilizes earthworms to change the form of heavy metals, thereby reducing their environmental risks. However, animal remediation technology is in a smaller scale [8].

Microbial remediation includes cell metabolism, absorption and transport of macromolecules on the surface, bio-adsorption, vacuolization, sedimentation, and redox reactions. Specifically, the bio-pile technology is to artificially intensify the contaminated soil piles, and therefore promoting the growth of indigenous micro-organisms or exogenous micro-organisms with the ability to degrade contaminants to degrade pollutants in the soil. It is suitable for treating biodegradable organics such as petroleum hydrocarbons. However, it is not suitable for the remediation of soils contaminated by heavy metals, organic pollutants that are difficult to degrade, and contaminated clay. Similarly, the in-situ bio-ventilation technology promotes the degradation of pollutants by supplying air or oxygen to the soil. At the same time, the utilization of pressure gradients in the soil promotes the flow of volatile organics and degradation products to the extraction wells, therefore, the VOCs could be extracted and removed. The effect of contaminant removal can be enhanced by injecting hot air, nutrient solution, and externally degrading microbial agents.

Phytoremediation is an environmental pollution remediation technology that using certain plants which can tolerate and hyper-enrich toxic elements and their co-existing microbial systems to remove pollutants. Phytoremediation is the general term for extracting, absorbing, decomposing, transforming, or fixing toxic, harmful pollutants in soils, sediments, sludge, or ground and groundwater by using plant. In the narrow sense, it is a method of using natural growth or genetic engineering cultivated plants to remediate pollutants. The core of this technology is that the concentration of pollutants gradually decreases. Phytoremediation is economically effective, low-cost, and has little disturbance to the environment. The resulting pollutant-enriched plants can be treated in a unified manner. Furthermore, pollutants can be recovered from these plants and can be used in long-term, with large-scale field applications.

\section{Development Trend}

\subsection{Water and soil combined treatment}

Soil and groundwater are an organic whole of the natural community in the environment. When vertical infiltration of rainwater and groundwater flows through contaminated soil, pollutants will enter the groundwater phase through dissolution and desorption. When contaminated groundwater flows through uncontaminated or rehabilitated soil, pollutants in the groundwater are transferred from the groundwater phase to the soil phase through the adsorption of the soil [9]. This interaction between soil and groundwater has led to a combination of soil and groundwater treatment, being particularly important in contaminated land restoration. Simply focusing on soil and neglecting groundwater will inevitably lead to secondary pollution.

Taking China's national conditions into account, although it is difficult to change the phenomenon of focusing on soil and neglecting groundwater in the restoration of contaminated land in the short term, the combined remediation of soil and groundwater will become a future trend.

However, due to difficult control, longer cycle length and better concealment of groundwater remediation, and a lack of corresponding technical specifications, in the process of actual polluting land survey and restoration, in order to save costs, the soil pollution is often emphasized but the attention to groundwater is often insufficient [10]. Taking patent application indicators as an example, the total number of current groundwater remediation patents in the past 10 years is only 500, far below the total amount of soil remediation projects (4000). Affected by the National Environmental Protection Policy such as 'the Action Plan for Prevention and Treatment of Water Pollution' and 'the Action Plan for Soil Pollution Prevention and Control', the number of patents applied each year has gradually increased in recent years, however, there are few patents for 
groundwater remediation. The number of groundwater patents applications in 2015 was 85, while the number of soil patent applications for the same period reached 952. The number of the groundwater patents was less than $1 / 10$ of the amount of soil patents.

In fact, the United States was similar at the beginning of contaminated land restoration. According to the Super Fund's yearly land restoration object statistics, the United States only paid attention to soil remediation at the earliest stage. With the deepening of the understanding of contaminated soil remediation, it began to attach importance to groundwater remediation and even the remediation of soil and groundwater. Now it realizes the systematic restoration and management of contaminated land, and the combined treatment of soil and groundwater has become the consensus in the industry of the pioneer country. For the Super Fund's remediation of 1468 contaminated lands during 1982-2011, about 14\% project was only for the soil remediation, while groundwater remediation and combined remediation of groundwater and soil accounted for $59 \%$.

According to the results of 'National Soil Pollution Survey', the overall pollution of the soil in the southern region is more serious compared with the north and will be the major soil remediation market in the future. At the same time, the groundwater level in the southern regions is generally shallow, and the total depth of the northern region has increased from east to west. In most regions, it is more than 6 meters. Therefore, the remediation of contaminated land in the northern region can sometimes only repair contaminated soils, regardless of groundwater. However, for the shallow groundwater depth in the south, ignoring groundwater remediation will lead to secondary pollution. Therefore, with the implementation of land pollution legislation by the state and the strict supervision, the guarantee of the continuity of the repair effect, and the emphasis on the integration remediation are imperative.

\subsection{Development Trends of Remediation Strategies Based on Risk Control}

Considering that soil and groundwater remediation is a complicated and long-term process, it is difficult for many lands to be completely restored in a short time. In response to this situation, in the 1990s, foreign countries gradually introduced " institutional control " as a method of remediation, also used legal and administrative methods (non-engineering) to protect human health and the environmental safety of the site to eliminate and prevent the risk and harm that land pollution may bring, so as to achieve the purpose of controlling the potential risks of contaminated land from the legislative using the management means. Common institutional control measures include restrictions on entering polluted areas, drilling restrictions, land use restrictions, and so on. Behind this type of institutional control is a prevention and control strategy with risk management as the core idea. Referring to the process of soil pollution prevention and control in various countries in the world, the developed countries have adopted this strategy in the end and penetrated them into the links of legislation, standard formulation, and selection of technical measures, thereby carrying out large-scale treatment and repair of contaminated soil.

In short, the risk-based remediation strategy is to organically combine traditional pollution source control technologies and engineering controls with institutional controls based on the specific conditions of contaminated land, thereby systematically reducing the risk of human exposure to hazardous land pollutants.

In the process of urban development and construction in China, due to the huge profits of real estate development, the polluted land will inevitably come into close contact with human beings in the later real estate development process. Therefore, the current method of remediation of polluted land is still based on engineering repair methods. However, the promulgation of the Action Plan for Soil Pollution Prevention and Control highlighted that the country's transition from the total amount of pollutants based on pollution control to pollution risk assessment. 


\subsection{Soil remediation from single to union}

In China, There are many kinds of pollutants in polluted land. Also, due to the various hydrogeological conditions, combined with compound pollution, and different regional economic development, the traditional single repair technology is often difficult to achieve the goal of restoration. The development of a synergistic joint soil remediation model that provides the most efficient and economical remediation plan for contaminated land is bound to become an industry trend [3].

\subsection{Trend from ex-situ to in-situ remediation}

Due to the rapid and efficient ex-situ remediation, China's soil remediation market will continue this trend in the short term. However, the cost of ex-situ remediation is high, especially for larger and deeper sites. Not only this ex-situ remediation has great damage to the environment, but also has the disadvantages of high operational risk [11]. However, the in-situ repair techniques are inexpensive and have little damage to the soil. As the repair technology continues to be mature, in-situ remediation technologies such as soil gas phase extraction, in-situ bioremediation and other technologies will become mainstream in the future [10].

Considering national conditions, the wide application of in-situ remediation technology is still an important obstacle in China. However, with the popularity of the PPP model and further diversification of funding sources in the future, the in-situ remediation mode will inevitably become a long-term trend. In fact, in the 'Catalogue of Encouraging Import Services (No. 2016047)', the consultation and implementation services for contaminated soil remediation and rehabilitation (restricted in-situ remediation) have been included in the list. This symbolizes that the nation has realized that the in-situ remediation technology is currently a domestic urgent need, nevertheless, the domestic service providers are still not able to satisfy this technology. It reflects the national strategy and policy orientation and is in line with the economic and social development trends and directions. In addition, with the improvement of technology and the development of rapid pollution-based land remediation technology based on equipment, it will also greatly reduce the in-situ restoration time, making in-situ remediation technology more feasible.

\subsection{Development of contaminated soil remediation technology using equipment}

The application of soil remediation technology depends on the support of repaired equipment and monitoring equipment to a large extent. Remediation technologies such as phytoremediation, microbial remediation, thermal desorption of organic contaminated soils, heavy metal contaminated soil leaching, solidification/stabilization, and environmental monitoring, all have to use equipment to achieve [3]. Therefore, the urgent need for rapid and effective equipment enables the land to be redeveloped and utilized quickly and efficiently. The development and application of rapid repaired technology of contaminated soil based on equipment is a development trend.

\section{References}

[1] Xu X, Jiang L. Industry Analysis and Employment Proposal on Soil Remediation in China[J]. Urban Roads Bridges \& Flood Control, 2015.

[2] Xue Z Y. Present situation, characteristics of soil pollution in China and some suggestions for soil remediation[J]. Modern Chemical Industry, 2014.

[3] Yongming L. Current research and development in soil remediation technologies[J]. Progress in Chemistry, 2009, 20(3):117-132.

[4] Eto T, Fujita K, Terakura S, et al. Current Status of Research and Development in Soil Remediation Technology[J]. 
[5] Jensen J, Pedersen M B. Ecological Risk Assessment of Contaminated Soil[M]// Reviews of Environmental Contamination and Toxicology. Springer New York, 2006:73-105.

[6] Zhang F, Ma L, Zhang Z, et al. Application of Chemical Reduction Technology to Remediation of Cr-Contaminated Soil[J]. Environmental Protection of Chemical Industry, 2012, 32(5):419-423.5

[7] Hua Z T, Xin-Gang L I, Hong S, et al. Remediation of oil contaminated soil by solvent extraction[J]. Modern Chemical Industry, 2013, 33(8):31-35.

[8] Song X, Lin N, Yin P H. Contaminated Site Remediation Industry in China: Current State and Future Trends[J]. Soils, 2015.

[9] Wang Y W, Shu-Peng L I, Kang S G, et al. ANALYSIS ON DEVELOPMENT STATUS OF INDUSTRIAL CONTAMINATED SITES REMEDIATION IN CHINA[J]. Environmental Engineering, 2017.

[10] Liao Xiaoyong, CHONG Zhongyi, YAN Xiulan, et al. Urban Industrial Contaminated Sites: a New Issue in the Field of Environmental Remediation in China [J]. Environmental Science, 2011, 32(3):784-794.

[11] Yan M A, Wang D, Zhu X U, et al. CURRENT SITUATION,PROBLEMS AND COUNTERMEASURES OF INDUSTRIAL CONTAMINATED SITES REMEDIATION IN BEIJING[J]. Environmental Engineering, 2017. 